\title{
Editorial
}

\section{Thin-Film Photovoltaics 2014}

\author{
Gaetano Di Marco, ${ }^{1}$ Giuseppe Calogero, ${ }^{1}$ Aldo Di Carlo, ${ }^{2}$ Salvatore Lombardo, ${ }^{3}$ \\ Leonardo Palmisano, ${ }^{4}$ and Olindo Isabella ${ }^{5}$ \\ ${ }^{1}$ Istituto per i Processi Chimico-Fisici (IPCF), CNR, Viale Ferdinando Stagno d'Alcontres 37, 98158 Messina, Italy \\ ${ }^{2}$ Centre for Hybrid and Organic Solar Energy (CHOSE), University of Rome “Tor Vergata", Via del Politecnico 1, 00133 Rome, Italy \\ ${ }^{3}$ Istituto per la Microelettronica e Microsistemi (IMM), CNR, Zona Industriale Ottava Strada 5, 95121 Catania, Italy \\ ${ }^{4}$ DEIM, Università degli Studi di Palermo, Viale delle Scienze, Edificio 6, 90128 Palermo, Italy \\ ${ }^{5}$ Photovoltaic Materials and Devices EEMCS Faculty, Delft University of Technology, Mekelweg 4, 2628 CD Delft, Netherlands
}

Correspondence should be addressed to Gaetano Di Marco; dimarco@its.me.cnr.it

Received 11 May 2015; Accepted 11 May 2015

Copyright (c) 2015 Gaetano Di Marco et al. This is an open access article distributed under the Creative Commons Attribution License, which permits unrestricted use, distribution, and reproduction in any medium, provided the original work is properly cited.

The interest toward alternative energy sources to fossil fuels (still the most convenient in terms of efficiency and cost) is current. Initiatives that seek to convert wind, geothermal energy, hydropower, marine, solar thermal energy, and photovoltaics or derived from biomass energy into other forms continue to involve several research groups. For example, the transfer from solar energy into electricity, which in turn can be used for water splitting and for the subsequent production of hydrogen, seems a major challenge to provide a sustainable contribution to the "Earth system" together with the realization of devices having high conversion efficiencies, low environmental impact, and low production costs. In our opinion the use of heterogeneous forms of available energy could definitely give a rational response to the growing global demand of energy, respecting the environment.

This special issue is following a series that began in 2010 . The aim is to provide a continuous update on state-of-the-art in the field of materials, nanotechnology, theoretical models, and technological aspects that belong to the science of thin film photovoltaics (TFPV). Despite this issue having a limited number of contributions, it is interesting to point out that the contribution provided by colleagues with their research have kept alive this initiative undertaken by this journal. Here is a timely synthesis of all contributions received which can stimulate the development of new ideas in the field of photovoltaic and highlight interest in this initiative in the future.
In "Graded Carrier Concentration Absorber Profile for High Efficiency CIGS Solar Cells," an innovative CIGSbased solar cells model with a graded doping concentration absorber profile is demonstrated, in order to achieve high efficiency values (up to 21\%). A depth-dependent study has shown that the proposed graded carrier concentration profile structures owns the same advantages of a CIGS cell having a $\mathrm{Ga}$ back graded profile, thanks to a quasielectrical field generated through the absorber and directed towards the back-contact. In addition, both the $E_{C}$ and the $E_{V}$ increase in the direction of the back-contact; that is, $E_{g}$ is constant along the depth. For this reason, the proposed cell structure overcomes the limitation of Ga back graded profile presenting a generation process that is definitely more efficient.

In "Enhancement of Spectral Response in $\mu \mathrm{c}-\mathrm{Si}_{1-x} \mathrm{Ge}_{x}$ :

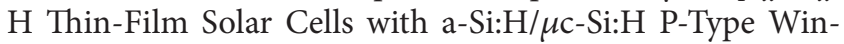
dow Layers", a technique to improve microcrystalline silicongermanium $\mathrm{p}-\mathrm{i}-\mathrm{n}$ single-junction thin-film solar cells deposited on textured $\mathrm{SnO}_{2}:$ F-coated glass substrates is studied. In fact, in thin film silicon solar cells deposited on glass, the prevalent technology is based on hydrogenated amorphous silicon. In this field hydrogenated microcrystalline silicon has attracted attention as alternative promising material, since compared to amorphous $\mathrm{Si}$ has a higher resistance to the Staebler-Wronski effect, a light-induced degradation which deteriorates the long-term efficiency, and a narrower bandgap which improves the near-infrared (NIR) response. As further 
improvement it has been shown that the addition of $\mathrm{Ge}$ into the microcrystalline Si network, that is, the use of a microcrystalline silicon germanium absorber, enhances even more the NIR spectral response. Finally, also the quality of the front transparent conducting oxide (TCO), generally textured $\mathrm{SnO}_{2}: \mathrm{F}$, has a significant influence on the cell performance. To form hydrogenated microcrystalline $\mathrm{Si}$, a gas mixture of $\mathrm{H}_{2}$ and $\mathrm{SiH}_{4}$ with a large $\mathrm{H}_{2}$ content is generally utilized, and the exposure to $\mathrm{H}_{2}$-containing plasma of the $\mathrm{SnO}_{2}: \mathrm{F}$ TCO is believed to produce $\mathrm{Sn}$ reduction which degrades solar cell performance. The effect worsens with the addition of $\mathrm{GeH}_{4}$ to form the hydrogenated microcrystalline $\mathrm{SiGe}$. In the contribution reported in this special issue it is shown that the performances of hydrogenated microcrystalline SiGe solar cells can be improved by using a novel structure based on a double p-type window layer made of hydrogenated amorphous Si and microcrystalline Si films.

In "Surface Photovoltage Spectroscopy and AFM Analysis of CIGSe Thin Film Solar Cells," the band gap, grain size, and topography of a $\mathrm{Cu}(\mathrm{In}, \mathrm{Ga}) \mathrm{Se}_{2}$ (CIGSe) thin film solar cell are analyzed using surface photovoltage spectroscopy (SPV) and atomic force microscopy (AFM) techniques. The sample investigated has been fabricated by a three-stage coevaporation process. The band gap energy of the CIGSe, $\mathrm{In}_{2} \mathrm{~S}_{3}$, and $\mathrm{ZnO}$ is deduced by extrapolation of the SPV signal slopes to the photon energy axis and found to be 1.1, 1.3 , and $2.6 \mathrm{eV}$, respectively. The AFM measurements yield information on orientation of the atomic nucleation and material homogeneity, founding grains very oriented with average size almost uniform $<1 \mu \mathrm{m}$. The topography presents a homogeneous diffusion of the $\operatorname{In}_{2} S_{3}$ or deionized water into the CIGSe layer after the annealing at $400^{\circ} \mathrm{C}$. These growth conditions have a strong influence on the surface which looks slightly rough (root mean square $37.8 \mathrm{~nm}$ ) almost smooth with no cracks.

In "Measurements and Simulations on the Mechanisms of Efficiency Losses in HIT Solar Cells," the optoelectrical behaviour of heterojunction solar cells was investigated by means of TCAD simulations and compared with that of conventional crystalline silicon solar cell. In this way the main problems affecting heterojunction devices could be identified. After measuring and simulating optimized structures from both technologies, two different losses mechanisms could be addressed into heterojunction devices. In particular, the presence of the amorphous silicon layer led to smaller distance between the metal lines causing higher shadowing and to a slight increase of reflectivity around the $600 \mathrm{~nm}$ wavelength. That is, a lower fill factor and a higher reflectivity for the heterojunction solar cell could be seen. The fill factor decrease is due to a higher potential loss along the $\mathrm{p}-\mathrm{n}$ junction that causes a higher diode current. Shrinking the pitch can prevent this shortcoming but causes a higher shadowing. In addition, the not ideal optical coupling between the amorphous silicon and the antireflective coating in the wavelength range from $400 \mathrm{~nm}$ to $600 \mathrm{~nm}$ causes lower short circuit current density. This effect could be reduced by reducing the thickness of the amorphous silicon layer; however it cannot be smaller than the value in use to prevent quantum effects.

In "Light Scattering and Current Enhancement for Microcrystalline Silicon Thin-Film Solar Cells on Aluminium-Induced Texture Glass Superstrates with Double Texture," some microcrystalline silicon $(\mu \mathrm{c}-\mathrm{Si}: \mathrm{H})$ thin solar cells are processed on glass superstrates having both micro- and nanoscale surface textures. The AIT glass superstrates employed in the solar cells are double textured and are prepared by conventional acid etching of the sputter-deposited transparent conductive oxide (TCO). The influence of the surface topology on the optical scattering behavior and on the $\mu \mathrm{c}$-Si:H film grown was investigated by means atomic force microscopy (AFM), diffuse scattering into air for visible light, and short circuit current enhancement measurements. Also the mean feature size of the textured superstrates is a good indicator for the mean lateral feature size of randomly textured surfaces and can be obtained from AFM image data processing. In order to investigate the benefit of this double texture different samples are used. The resulting double texture has much higher surface roughness (RMS) than the reference sample. As expected, from this study the current enhancement is mainly ascribed to the better light absorption for the infrared light since the haze values in the long-wavelength region improved significantly by using AIT1 and AT2 glass superstrates. However a local shunt formation also emerged, due to defective areas (cracks) formed when the $(\mu \mathrm{c}-\mathrm{Si}: \mathrm{H})$ films are deposited on AIT glass superstrates above the deep valleys of the microtextured kinks decreasing the solar cells efficiency. Further optimization still needs to be done in order to decrease the shunting effect while maintaining the light scattering abilities of the superstrates.

In "Optical Characterization of Different Thin Film Module Technologies," a combination of fast and nondestructive methods for a complete quality control of three types of thin film solar module technologies such as a-SI, CdTe, and CIS is investigated. The authors propose camera-based measurements using electroluminescence (EL), photoluminescence (PL), and infrared (IR) technologies, with the aim to increase the numbers of detectable defects and to determine their origin. By using the thermal behavior of cells in a module it is possible to see short circuits, shunts, inactive cell parts, moisture, and defective bypass diodes. With the Dark-lock-in thermography a pulsed current is applied to the solar device without illumination and when the current applied as dark current flows in the solar device causes heating which can be detected and analyzed by a cooled microbolometer based IRcamera. EL measurements take advantages of the radiative interband recombination of the excited charge carriers in the solar devices. The light emitted signal is detected by a Si-CCD-camera (range $300 \mathrm{~nm}-1000 \mathrm{~nm}$ ). This method is a useful tool for investigating electrical nonhomogeneities caused by intrinsic defects. PL measurements can be used to detect defects throughout the entire solar cell manufacturing process. PL-images help to find out if material defects or contacting problems are the reason for power losses in 
solar cells in modules. Anyway according to this study the combination of all these techniques is necessary to identify as many defects as possible in nondestructive way.

Gaetano Di Marco Giuseppe Calogero Aldo Di Carlo Salvatore Lombardo Leonardo Palmisano Olindo Isabella 

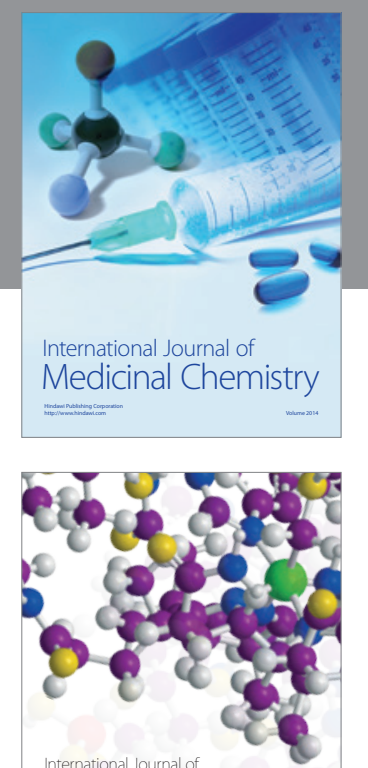

\section{Carbohydrate} Chemistry

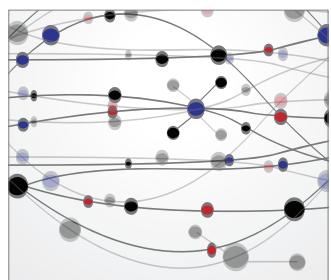

The Scientific World Journal
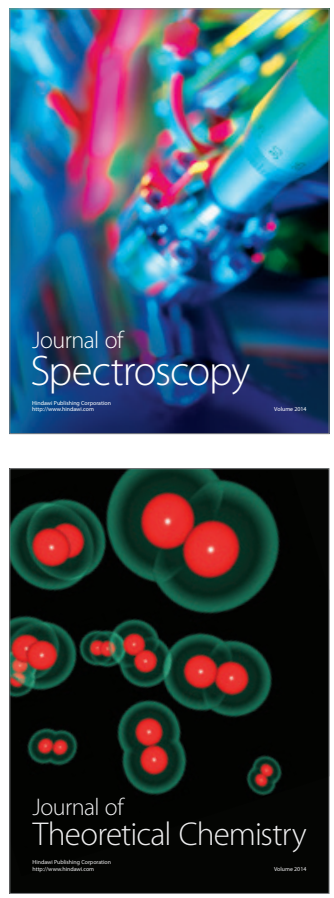
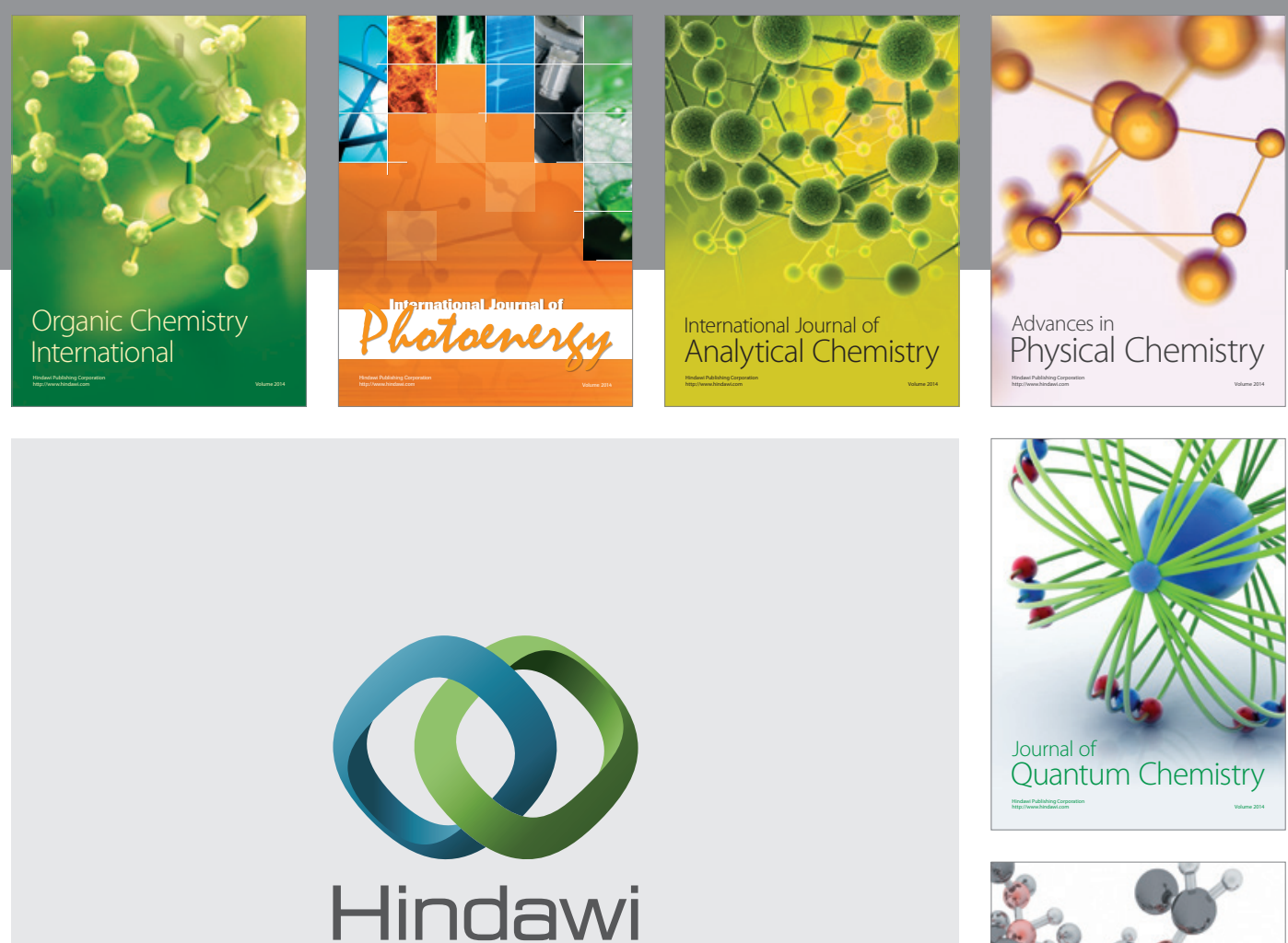

Submit your manuscripts at

http://www.hindawi.com

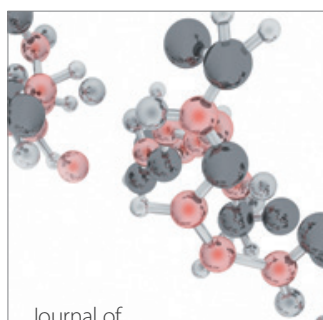

Analytical Methods

in Chemistry

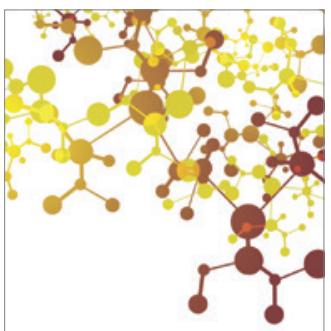

Journal of

Applied Chemistry

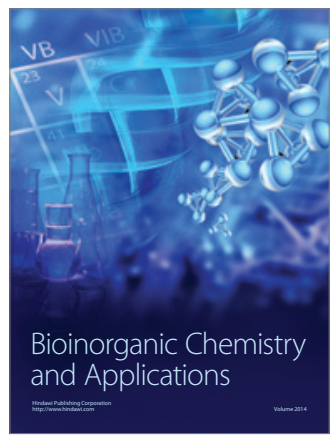

Inorganic Chemistry
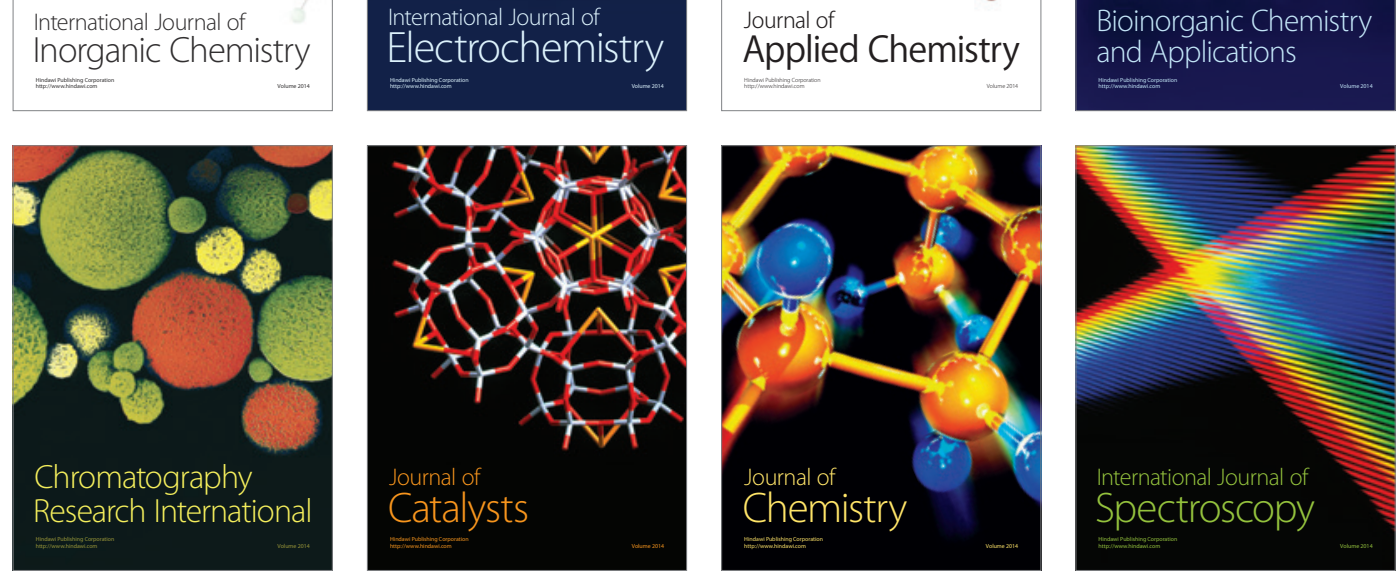